\title{
HvCEBiP, a gene homologous to rice chitin receptor CEBiP, contributes to basal resistance of barley to Magnaporthe oryzae
}

\author{
Shigeyuki Tanaka ${ }^{1,6}$, Akari Ichikawa $^{1}$, Kaori Yamada', Gento Tsuji', Takumi Nishiuchi', Masashi Mori ${ }^{3}$, Hironori Koga ${ }^{3}$
} , Yoko Nishizawa ${ }^{4}$, Richard O'Connell ${ }^{5}$, Yasuyuki Kubo ${ }^{1 *}$

\begin{abstract}
Background: Rice CEBiP recognizes chitin oligosaccharides on the fungal cell surface or released into the plant apoplast, leading to the expression of plant disease resistance against fungal infection. However, it has not yet been reported whether CEBiP is actually required for restricting the growth of fungal pathogens. Here we evaluated the involvement of a putative chitin receptor gene in the basal resistance of barley to the ssd1 mutant of Magnaporthe oryzae, which induces multiple host defense responses.

Results: The mossd1 mutant showed attenuated pathogenicity on barley and appressorial penetration was restricted by the formation of callose papillae at attempted entry sites. When conidial suspensions of mossd1 mutant were spotted onto the leaves of HvCEBiP-silenced plants, small brown necrotic flecks or blast lesions were produced but these lesions did not expand beyond the inoculation site. Wild-type M. oryzae also produced slightly more severe symptoms on the leaves of HvCEBiP-silenced plants. Cytological observation revealed that these lesions resulted from appressorium-mediated penetration into plant epidermal cells.

Conclusions: These results suggest that HVCEBiP is involved in basal resistance against appressorium-mediated infection and that basal resistance might be triggered by the recognition of chitin oligosaccharides derived from M. oryzae.
\end{abstract}

\section{Background}

To resist attack by microbial pathogens, plants have evolved to recognize them, triggering the expression of diverse defense reactions. The currently accepted model is that plants recognize conserved pathogen-associated molecular patterns (PAMPs) through corresponding pattern recognition receptors (PRRs) which in turn trigger plant immune responses [1-3]. The involvement of PRRs in disease resistance against bacterial pathogens is welldocumented. For example, the $\mathrm{N}$-terminal amino acid sequence of bacterial flagellin (designated as flg22) can be recognized through the corresponding receptor FLS2 in Arabidopsis thaliana [4,5]. In addition, the N-terminal sequence of bacterial translational elongation factor $\mathrm{Tu}$

\footnotetext{
* Correspondence: y_kubo@kpu.ac.jp

'Laboratory of Plant Pathology, Graduate School of Life and Environmental

Sciences, Kyoto Prefectural University, Kyoto 606-8522, Japan

Full list of author information is available at the end of the article
}

(designated as elf18) can be recognized through the corresponding receptor EFR [6,7].

In contrast to bacterial PAMP receptors, much less is known about the role of fungal PAMP receptors in plants. It is conceivable that oligosaccharides derived from chitin or glucan may function as PAMPs because they are major structural components of fungal cell walls and can induce the expression of several defenserelated genes when they are applied to plants $[8,9]$. The rice plasma membrane glycoprotein CEBiP (Chitin Elicitor Binding Protein) was shown to be an important component for chitin-derived signaling and is thought to be a receptor for fungal PAMPs [10]. CEBiP was identified as a chitin-binding protein from suspension cultured rice cells and contains two LysM (lysin) domains which mediate binding to oligosaccharides. Physiological experiments suggest that $\mathrm{CEBiP}$ is required for the production of reactive oxygen species by rice plants in response to treatment with chitin elicitor [10].
Ciomed Central

() 2010 Tanaka et al; licensee BioMed Central Ltd. This is an Open Access article distributed under the terms of the Creative Commons Attribution License (http://creativecommons.org/licenses/by/2.0), which permits unrestricted use, distribution, and reproduction in any medium, provided the original work is properly cited. 
It is assumed that $\mathrm{CEBiP}$ recognizes chitin oligosaccharides present on the fungal cell surface or released into the plant apoplast, leading to the expression of plant disease resistance against fungal infection. However, it has not yet been reported whether CEBiP is actually required for restricting the growth of fungal pathogens in rice.

Magnaporthe oryzae is an ascomycete fungus that causes the devastating blast disease in rice [11]. In the previous report, we have generated $s s d 1$ mutants in $M$. oryzae and the cucumber anthracnose fungus Colletotrichum orbiculare, in which infection of their respective host plants was restricted by cellular defense responses [12]. Subsequently, by inoculating the C. orbiculare ssd 1 mutant onto Nicotiana benthamiana plants in which defense-related genes were silenced, we evaluated the involvement of those genes in basal defense. These experiments revealed that plants in which genes encoding specific MAPKK (MEK2) and MAPKs (SIPK/WIPK) had been silenced were susceptible to the $s s d 1$ mutant, as well as the wild-type strain [13]. Furthermore, we revealed that these MAPKs were activated by fungal cell surface components during infection and that the level of MAPK activation induced by the $s s d 1$ mutant was higher than by the wild-type strain, suggesting that MAPK signaling is required for enhanced basal defense and restriction of fungal infection. In addition, use of the $s s d 1$ mutant together with gene-silenced plants allowed us to critically evaluate the involvement of specific defense-related genes in basal resistance by assessing whether the $s s d 1$ mutant could produce disease lesions on the silenced plants.

In plants, RNA interference (RNAi) is a powerful tool for the evaluation of gene function [14]. For RNAi, it is necessary to generate transgenic plants that express a partial fragment of the target gene, but considerable time is required to obtain seeds from $\mathrm{T}_{1}$ transformants. In contrast, virus-induced gene silencing (VIGS) is a simple, rapid method to transiently generate knockdown plants that avoids the need for stable transformation [15]. Although procedures for VIGS are not yet established for rice, there are reports that VIGS is applicable to barley through the use of barley stripe mosaic virus (BSMV) [16,17]. Barley is a susceptible host plant for $M$. oryzae, so that interactions between $M$. oryzae and barley provide a model for the molecular analysis of compatible interactions between monocot plants and fungal pathogens [18].

In this study, we have exploited the barley-Magnaporthe pathosystem to evaluate the involvement in basal resistance of genes encoding a putative PAMP receptor, namely $H v C E B i P$, which is homologous to the rice chitin receptor CEBiP. For this, we used the $M$. oryzae ssd 1 mutant and BSMV-mediated gene silencing. We present evidence that $H \nu C E B i P$ contributes to basal defense against appressorium-mediated infection by $M$. oryzae in barley.

\section{Results \\ Magnaporthe oryzae SSD1 is required for infection of barley}

In previous work we showed that the SSD1 gene of $M$. oryzae is essential for the successful infection of susceptible rice plants, and that the failure of mossd 1 mutants to infect was associated with the accumulation of reactive oxygen species (ROS) by host cells [12]. First, we examined whether the SSD1 gene is also essential for the infection of barley (Hordeum vulgare). When conidial suspensions of the wild-type strain Hoku-1 were inoculated onto leaves, necrotic lesions similar to those of rice blast disease could be observed at 4 days post inoculation (dpi). In contrast, leaves inoculated with the mossd 1 mutants $\mathrm{K} 1$ and $\mathrm{K} 4$ did not show visible disease symptoms (Figure 1A). When conidial suspensions were spotted onto intact leaf blades of barley, mutant K1 did not produce any disease symptoms, although the wildtype Hoku-1 forms typical blast lesions at inoculation sites at $4 \mathrm{dpi}$ (Figure 1B). To test whether the K1 mutant retained invasive growth ability, conidial suspensions were spotted onto wound sites on the surface of barley leaves. The mutant produced brown necrotic flecks at wound sites but disease symptoms did not spread further, in contrast to the wild-type Hoku-1 which could form typical blast lesions after infection through wounds (Figure 1B). Overall, the pathogenicity of the $M$. oryzae ssd1 mutants was severely attenuated on barley, producing an infection phenotype similar to that seen previously on rice [12].

Microscopic analysis showed that the mossd 1 mutant formed appressoria on the plant surface indistinguishable from those of the wild-type strain Hoku-1 (Figure 2A). However, while Hoku-1 produced intracellular infection hyphae inside host epidermal cells, mutant $\mathrm{K} 1$ had formed no infection hyphae at $48 \mathrm{hpi}$ (Figure 2A). To observe the responses of $H$. vulgare cells to attempted infection by the mutant, inoculated leaves were stained with 3,3'-diaminobenzidine (DAB) to detect $\mathrm{H}_{2} \mathrm{O}_{2}$ accumulation. However, no significant accumulation of $\mathrm{H}_{2} \mathrm{O}_{2}$ was detectable in host cells after inoculation with Hoku-1 or K1 at 48 hpi (data not shown). Next, we attempted to detect the formation of autofluorescent papillae under appressoria using epi-fluoresence microscopy [18]. At sites of attempted penetration by the mossd 1 mutant, autofluorescent papilla-like structures could be observed beneath approximately $80-90 \%$ of mutant appressoria (Figure 2B), and intracellular infection hyphae were only rarely observed inside host cells (Figure 2C). On the other hand, the frequency of papilla formation under appressoria of Hoku-1 was only $20 \%$ 


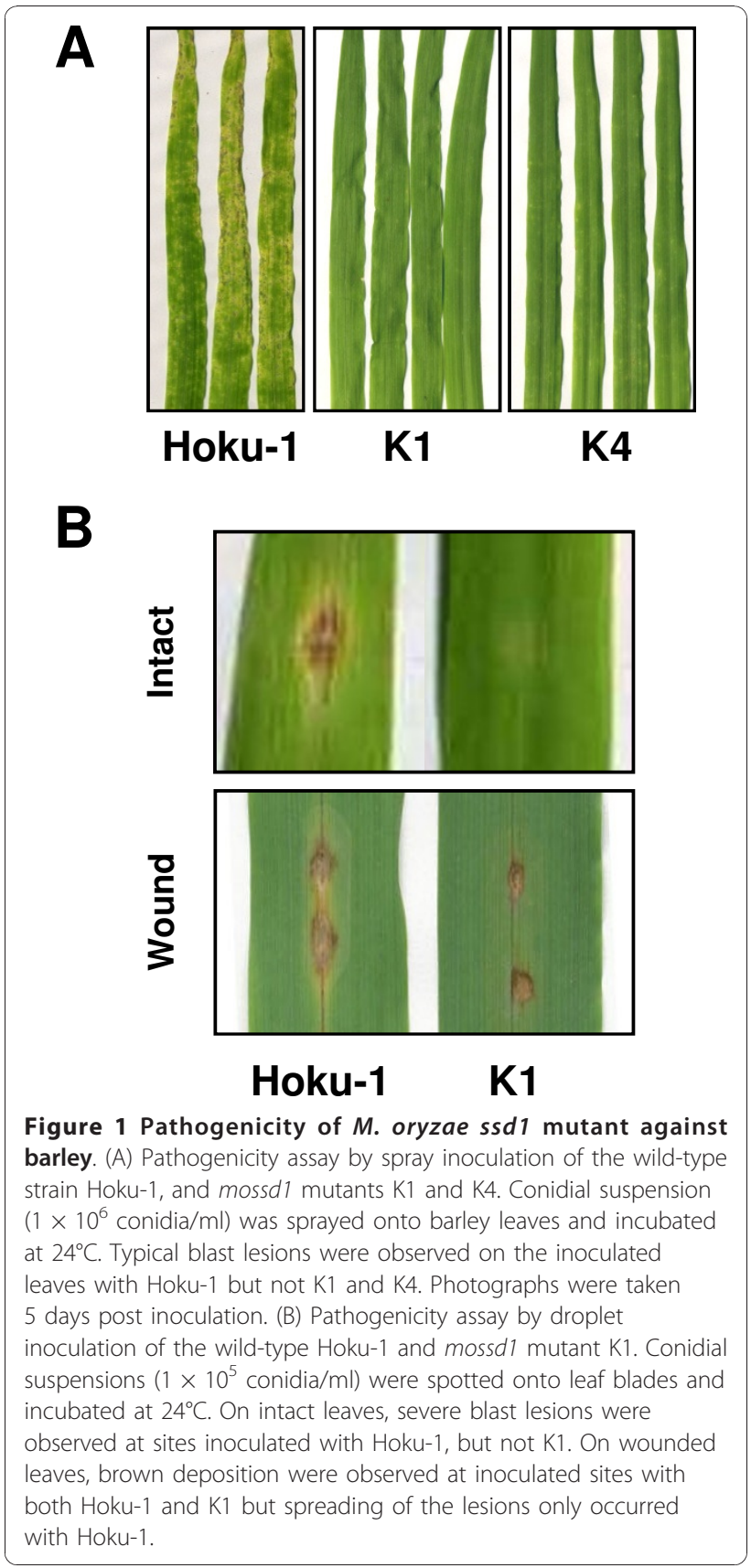

and infection hyphae developed from $60 \%$ of appressoria (Figure 2C). These results suggest that the localized deposition of cell wall material (papillae) at attempted fungal entry sites forms part of the basal defense response of barley epidermal cells to appressorial penetration by $M$. oryzae.

\section{Virus-induced gene silencing of HvCEBiP using barley} stripe mosaic virus

Chitin is major structural component of fungal cell walls and is therefore likely to function as a PAMP [10]. We
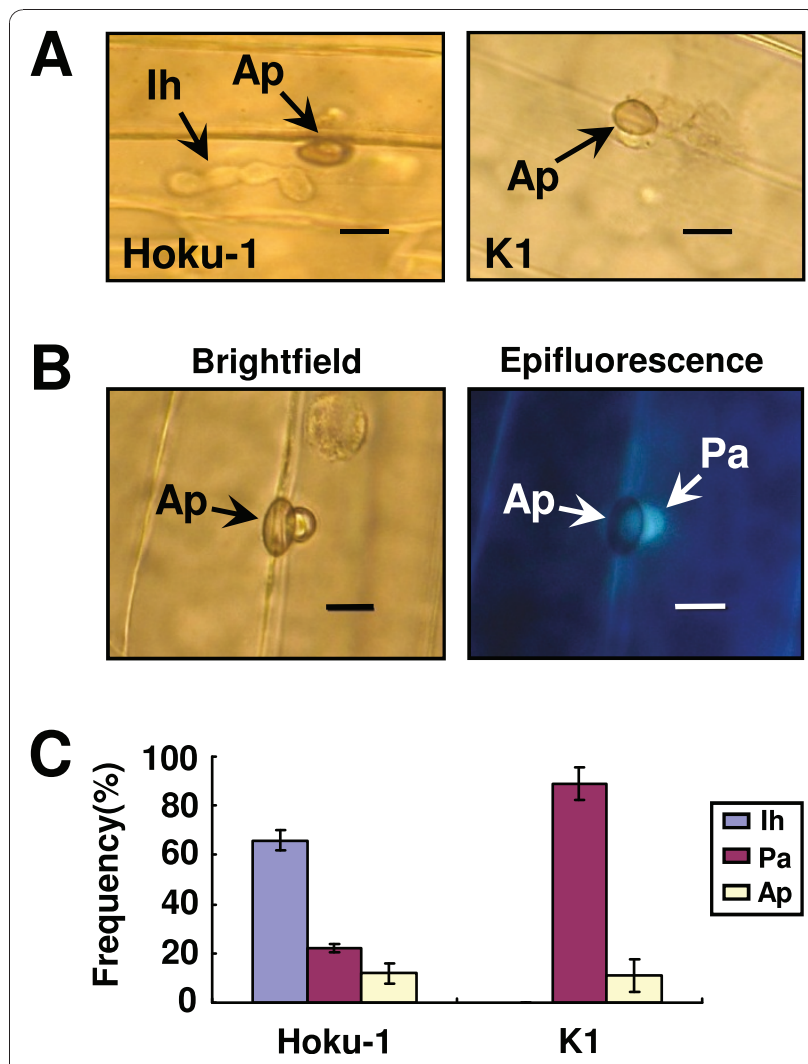

Figure 2 Cytology of infection of barley leaf tissue by the $M$. oryzae ssd1 mutant. (A) Infection phenotypes of the wild-type Hoku-1 and mossd1 mutant K1. Inoculated leaves at 48 hpi were decolorized and observed with light microscopy. The wild-type strain Hoku-1 formed infection hyphae from appressoria on the plant surface but mossd1 mutant K1 did not show infection hyphae inside plant cell. Ap, appressorium; Ih, infection hypha; Bar $=5 \mu \mathrm{m}$. (B) Formation of papilla-like structures under appressoria of $s s d 1$ mutant K1. At 48 hpi, the decolorized leaves were observed with epi-fluorescence microscopy. Autofluorescent papillae were visible beneath appressoria. Ap, appressorium; Pa, papilla; $\mathrm{Bar}=5 \mu \mathrm{m}$. (C) Frequency of appressorial penetration and host papilla formation. Leaves sprayed with conidial suspension $\left(1 \times 10^{6}\right.$ conidia/ml) were observed at $48 \mathrm{hpi}$. Infection phenotypes were classified as follows; Ih, infection hyphae under appressoria; Pa, papilla under appressoria; Ap, appressoria without papillae or infection hyphae. Appressoria of the wild-type strain Hoku-1 penetrated with high frequency to form infection hyphae, but those of ssd1 mutant K1 induced papillae with high frequency.

therefore searched for a gene homologous to the CEBiP chitin receptor of rice using a barley EST database (TIGR plant transcript assemblies; http://blast.jcvi.org/ euk-blast/plantta_blast.cgi) and found an assembled sequence TA30910_4513 which contains the putative full-length coding sequence. The predicted amino acid sequence showed $66 \%$ identity to rice CEBiP. Furthermore, this sequence contained a signal peptide at the $\mathrm{N}$-terminus, and two LysM motifs and a transmembrane region in the $\mathrm{C}$-terminal region, which are all present in 


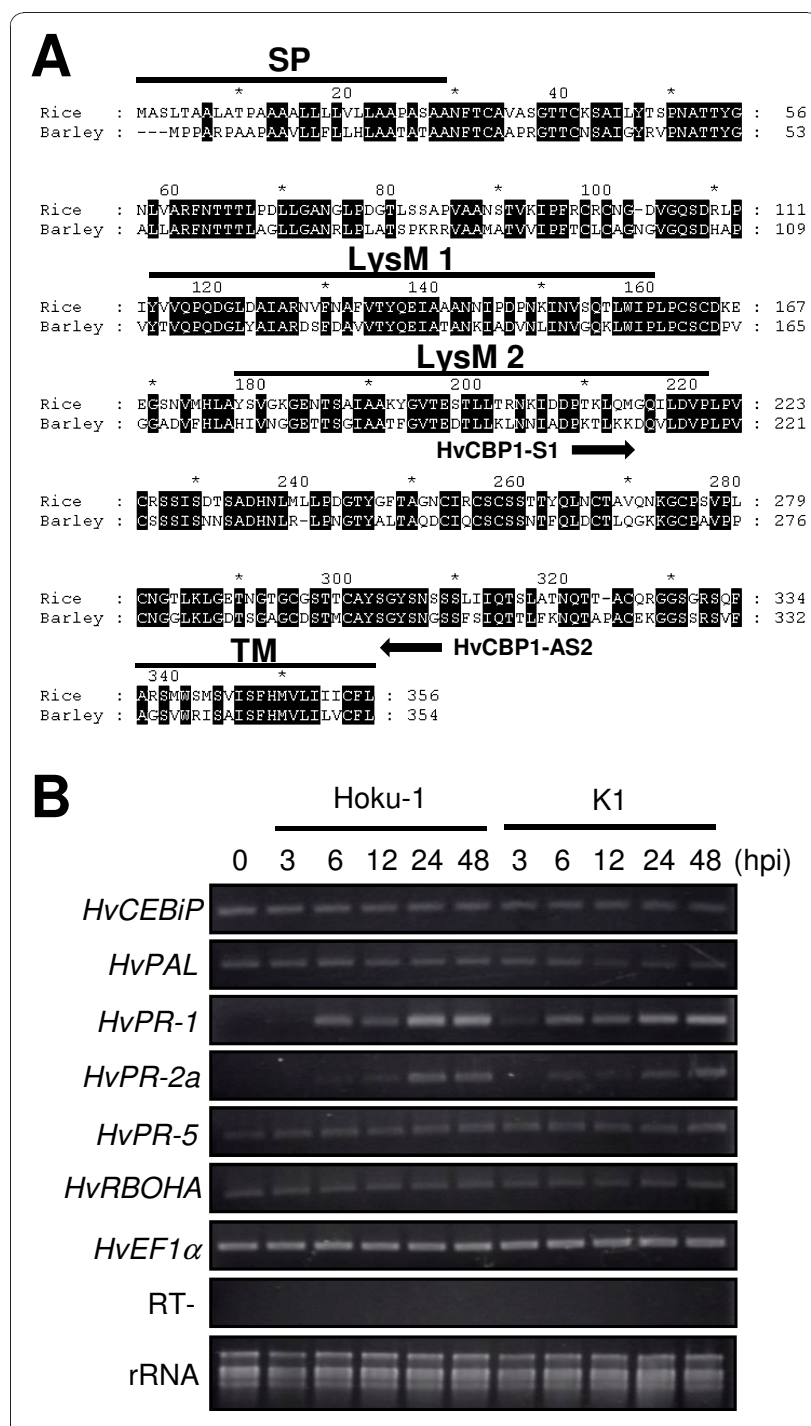

Figure 3 Sequence and expression profiling of HvCEBiP. (A) Alignment of the amino acid sequences between rice CEBiP (Rice) and barley HvCEBiP (Barley). Putative coding sequence of HvCEBiP was aligned with rice CEBiP. Identical amino acids are highlighted with black boxes. SP, signal peptide; LysM 1/LysM 2, LysM motif; TM, transmembrane region. Arrows indicated primer position used for gene silencing of HvCEBiP. (B) Expression profiling of HvCEBiP and several defense-related genes. Conidial suspensions $\left(1 \times 10^{5}\right.$ conidia/ml) of the wild-type strain Hoku-1 or mossd1 mutant K1 were spotted onto barley leaves and total RNAs were extracted from inoculated tissues at 0 (no inoculation), 3, 6, 12, 24 and 48 hpi for RT-PCR. The expression of HvCEBiP was detectable with similar transcript levels at all time points in the leaves inoculated with either Hoku-1 or K1. The expression of HVPAL, HVRBOHA and HVPR-5 was detectable at all time points, but expression of HVPR-1 and HvPR-2a was induced after inoculation with M. oryzae. For checking genomic contamination, PCR of HvEF1 $\alpha$ was performed using total RNA as template (designated as RT-). Ribosomal RNAs are presented as loading control. rice CEBiP (Figure 3A). Therefore, we consider this gene is very likely to be orthologous to rice CEBiP, and accordingly designated the gene $H v C E B i P$. When we examined the expression of $H \nu C E B i P$ during the course of infection of barley by $M$. oryzae (Figure 3B), transcripts were detectable at all time points $(3,6,12,24$, $48 \mathrm{hpi}$ ), indicating that $H \nu C E B i P$ is likely to be constitutively expressed in barley. In addition, we also examined the expression of selected defense-related genes during infection. Genes homologous to phenylalanine ammonia lyase, respiratory burst oxidase homologue $\mathrm{A}$ and pathogenesis-related proteins 1,2 , and 5 were searched from the barley EST database, and designated as $H v P A L$, $H v R B O H A, H v P R-1, H v P R-2 a$ and $H v P R-5$, respectively. As shown in Figure $3 \mathrm{C}$, transcripts of $H v P A L$, $H v R B O H A$ and $H v P R-5$ could be detected at all time points, suggesting they are constitutively expressed. However, it should be noted that both $P A L$ and $P R 5$ generally belong to multi-gene families and we cannot exclude that gene members other than those evaluated in this experiment may be inducible by fungal infection. $H v P R-1$ and $H v P R-2 a$ expression could not be detected at 0 hpi (no inoculation) but was detected from $6 \mathrm{hpi}$, suggesting the expression of $H v P R-1$ and $H v P R-2 a$ was induced by inoculation with $M$. oryzae. However, there were no major differences in plant defense gene expression induced by the wild type and mossd1 mutant K1.

Next, to evaluate the involvement of $H \nu C E B i P$ in basal resistance of barley, we attempted to perform virusinduced gene silencing (VIGS) using the barley stripe mosaic virus (BSMV) [17]. Before silencing HvCEBiP, we first confirmed the efficiency of BSMV-mediated gene silencing in barley by silencing a gene encoding phytoene desaturase (PDS). After BSMV:PDS genomic RNA was inoculated into the first developed leaves of barley plants, a photobleaching phenotype typical of PDS deficiency was visible on the third developed leaves of all inoculated plants, indicating that BSMV-mediated gene silencing of PDS was effective in barley (see Additional file 1: Figure S1). For silencing of $H \nu C E B i P$, we first amplified a 298 bp partial fragment of $H \nu C E B i P$ from barley leaf cDNA and introduced it into plasmid pSL038-1 which carries the $\gamma$ genome of BSMV. The resulting construct, in which a fragment of the target gene is introduced in the antisense orientation, was designated as $\mathrm{p} \gamma: \mathrm{HvCEBiPas}$ (Figure 4A). The sequence used for silencing $H \nu C E B i P$ did not contain either of the two LysM motifs (Figure 3A). In the EST data base background, we selected unique sequences to HvCEBiP, although without access to the complete barley genome, we could not exclude that there might be other 


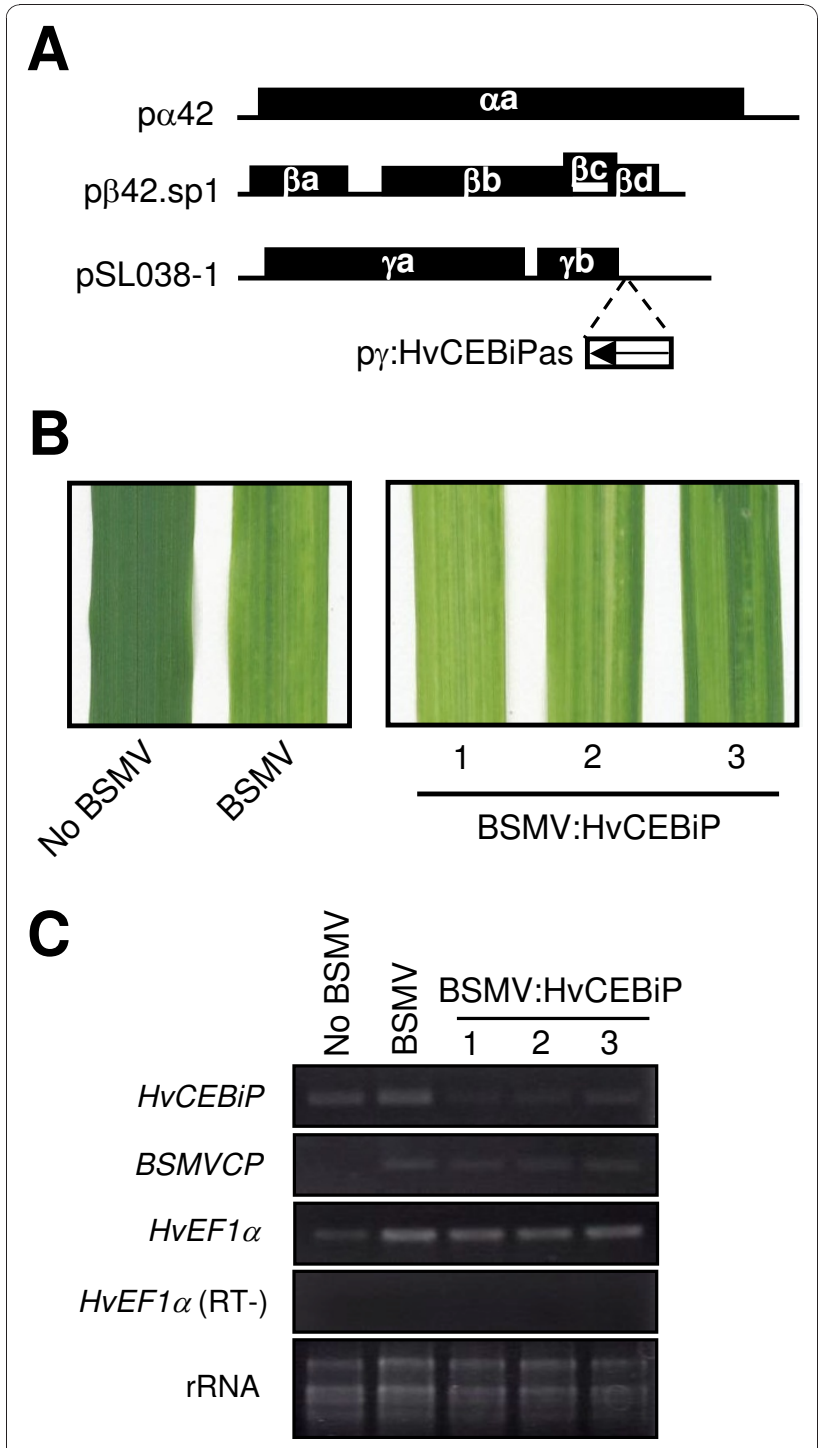

Figure 4 Evaluation of HvCEBiP gene silencing. (A) The genomic organization of BSMV and corresponding silencing constructs. Genomic RNA of BSMV was transcribed in vitro from pa42, p $\beta 42 . s p 1$ and PSL038-1, carrying the $\alpha, \beta$ and $\gamma$ genomes, respectively. Genomic RNA of BSMV:HvCEBiP was from p $\alpha 42, p \beta 42 . s p 1$ and $p \gamma$ : HvCEBiPas, which harbours a partial fragment of HvCEBiP in the antisense orientation. (B) The third-developed leaves of barley plants at 10 days after inoculation with BSMV genomic RNA onto the first leaves. Stripe mosaic symptoms were observed in the third leaves of BSMV- or BSMV:HVCEBiP-treated plants but not in untreated plants (No BSMV). (C) Evaluation of the silencing effect by RT-PCR. Total RNAs were extracted from the leaves shown in $\mathbf{B}$ and used for RT-PCR. BSMVCP encoding viral coat protein was detectable in BSMV- or BSMV:HvCEBiP-treated plants but not in untreated plant (No BSMV). The expression level of HVCEBiP was down-regulated in the third leaves of BSMV:HvCEBiP-treated plants compared to a BSMV-treated plant or untreated plant. For checking genomic contamination, PCR of HvEF1 $\alpha$ was performed using total RNA as template (RT-). Ribosomal RNAs are presented as loading control. potential CEBiP homologs that are silenced. Next, we attempted to evaluate the silencing effect of $H \nu C E B i P$ by RT-PCR. After inoculation of BSMV:HvCEBiP onto first-developed barley leaves, total RNA was extracted from the third-developed leaves and used for reverse transcription. Typical viral disease symptoms were observed in the third leaves of plants treated with BSMV (control) or BSMV:HvCEBiP genomic RNA (Figure 4B). In these leaves, the expression of both $B S M V C P$, encoding the BSMV coat protein, and $H v E F 1 \alpha$, encoding barley translational elongation factor, was detectable (Figure 4C). On the other hand, the third leaves of plants treated with BSMV:HvCEBiP showed reduced transcription levels of $H \nu C E B i P$ compared to control plants treated with BSMV (Figure 4C). These results indicate that the transcript level of $H \nu C E B i P$ was down-regulated by BSMV:HvCEBiP-mediated gene silencing in barley.

\section{HvCEBiP contributes to restricting infection by mossd 1 mutants}

To examine whether HvCEBiP is involved in the basal resistance of barley to Magnaporthe, we inoculated the mossd 1 mutant $\mathrm{K} 4$ onto the third-developed leaves of barley plants after inoculation of BSMV:HvCEBiP onto the first-developed leaves. To quantify the severity of disease symptoms produced by the mossd 1 mutant, we classified disease symptoms as follows; Type I, no visible symptoms; Type II, brown necrotic flecks; Type III, blast lesions without brown necrotic flecks (Figure 5A). On the leaves of BSMV-treated plants, most symptoms produced by mossd 1 mutant $\mathrm{K} 4$ were classified as Type I (Figure 5B), whereas on leaves of BSMV:HvCEBiP-treated plants Type II symptoms were produced at approximately half of the sites inoculated with $\mathrm{K} 4$ (Figure 5B). This tendency was confirmed in three independent experiments. When the wild-type strain Hoku1 was inoculated onto leaves of BSMV:HvCEBiP-treated plants, the frequency of Type III symptoms was slightly but consistently higher compared to the control plant, although these effects were not statistically significant (Figure 5B). When conidial suspensions were inoculated onto wound sites on the leaves of BSMV:HvCEBiP-treated plants, there was no significant difference in disease symptoms produced by Hoku-1 and K4 (data not shown), suggesting that the silencing of $H \nu C E B i P$ does not affect invasive growth ability through wounds. Taken together, these results suggest that HvCEBiP is involved in basal defense responses of susceptible barley plants to appressorial penetration by $M$. oryzae.

To determine whether the mossd 1 mutant was able to develop infection hyphae and colonize barley tissues, we 


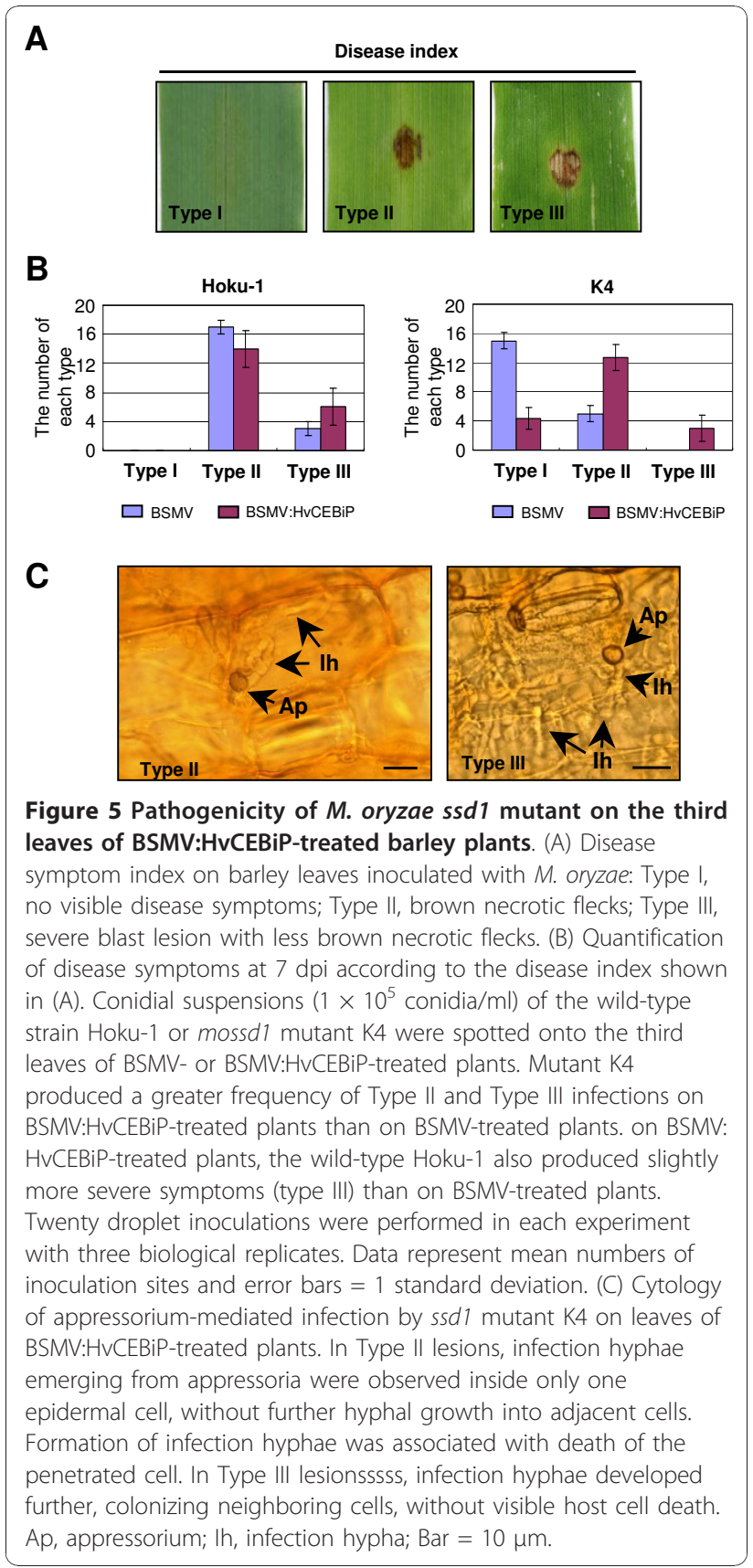

observed leaf inoculation sites in BSMV:HvCEBiP-treated plants at $96 \mathrm{hpi}$. At sites showing brown necrotic flecks (Type II symptom), appressoria were present on the leaf surface, and infection hyphae developed from appressoria inside the initially infected epidermal cell, which appeared to undergo a cell death reaction (Figure 5C). However, when we observed inoculation sites at $7 \mathrm{dpi}$, fungal hyphae had not colonized the neighboring host cells and hyphae were entirely confined to the first infected cell (data not shown). These observations suggest that mossd 1 mutant appressoria could penetrate into $H \nu C E B i P$-silenced plants but subsequent growth of the infection hyphae became restricted by host defense responses. However, at the few inoculation sites showing severe lesions (Type III), infection hyphae were seen to develop from appressoria without visible host cell death (Figure 5C). Taken together, these results suggest that $H \nu C E B i P$ contributes to host defense responses expressed after invasion of epidermal cells by $M$. oryzae infection hyphae.

To evaluate whether HvCEBiP is also involved in nonhost resistance, we inoculated conidia of the nonadapted maize anthracnose pathogen C. graminicola onto the third leaves of BSMV:HvCEBiP-treated plants. Although C. graminicola formed appressoria on the leaves of both BSMV - and BSMV:HvCEBiP-treated plants, intracellular infection hyphae were not observed, and no disease symptoms were produced (Figure 6). This suggests that $H \nu C E B i P$ does not play a critical role in resistance to non-adapted pathogens such as $C$. graminicola.

Next, we evaluated the possible role in basal defense of selected barley genes required for penetration

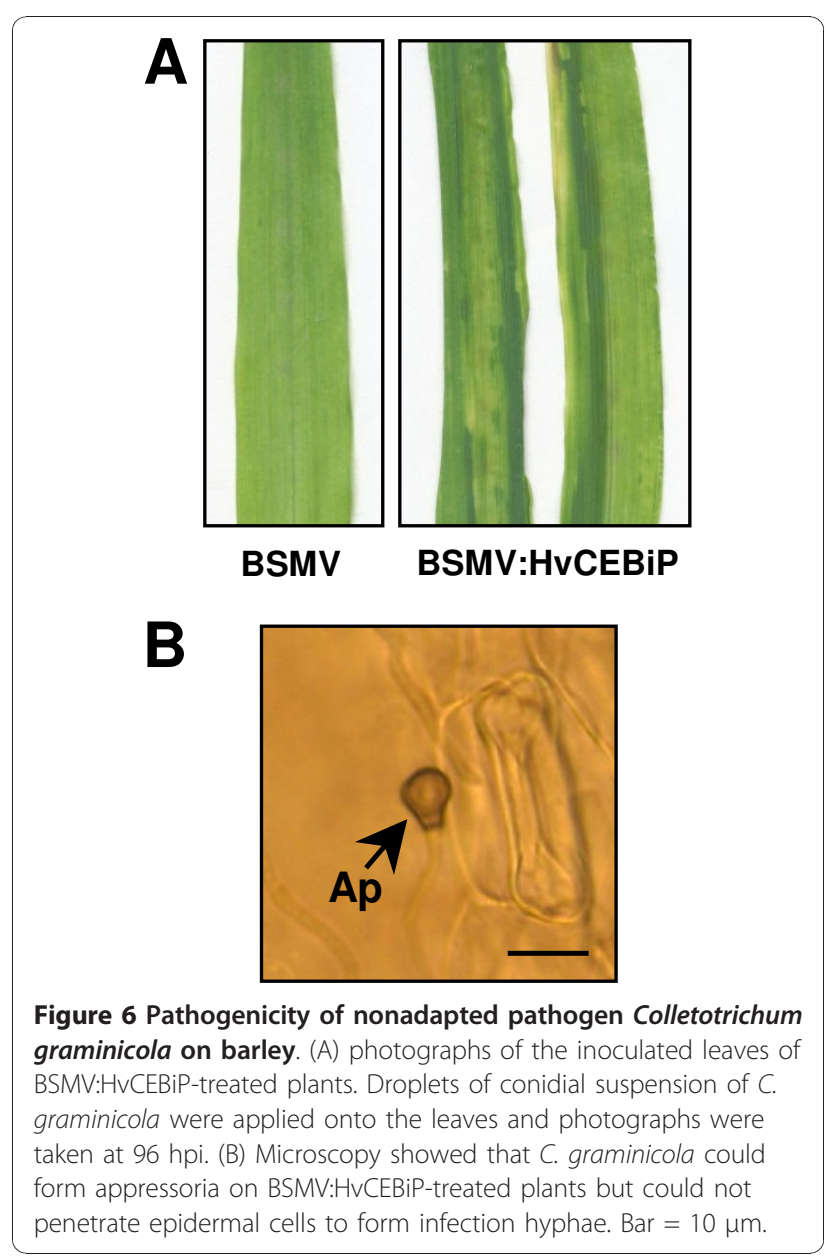




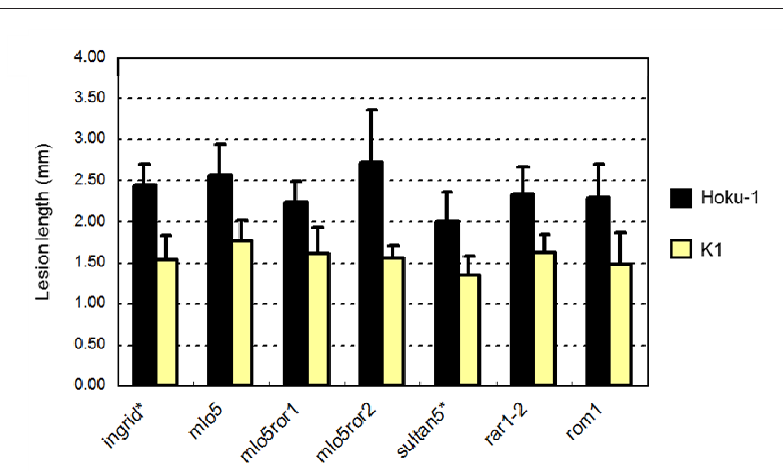

Figure 7 Pathogenicity test of the wild-type Hoku-1 and mossd 1 mutant $\mathrm{K} 1$ on a range of barley mutants affected in various defense-related genes. Droplets of conidial suspension were applied onto leaves of genetic mutants of mlo5, Ror1, Ror2, Rarl and Rom 1. Ingrid is the wild-type cultivar for mlo5, rorl and ror2 mutants. Sultan5 is the wild-type cultivar for rarl and rom 1.

resistance and $R$-gene mediated resistance to the powdery mildew fungus, Blumeria graminis f. sp. hordei. For this, we used barley mutant lines deficient in Ror 1 and Ror2 (required for mlo-specified resistance) [19,20], Rar1 (required for Mla12 resistance) [21] and Rom1 (restoration of Mla12-specified resistance) [22]. After inoculating conidial suspension of mossd1 mutant K4 onto leaves of these barley mutants, no significant differences in symptom severity were observed compared to the respective wild-type barley cultivars (Figure 7). It therefore appears that none of these genes are involved in restricting infection by the moss 11 mutant.

\section{Expression profiling of defense-related genes in HvCEBiP- silenced plants}

To identify plant defense-related genes that may be regulated by $\mathrm{HvCEBiP}$-mediated signaling, we evaluated the expression patterns of selected barley defense genes in the leaves of BSMV:HvCEBiP-treated plants (Figure 8). Total RNAs were extracted at $0 \mathrm{~h}$ (no inoculation), 24 $\mathrm{h}$ and $48 \mathrm{~h}$ after inoculation of the wild-type Hoku-1 or mossd 1 mutant K4 onto leaves of BSMV- or BSMV: HvCEBiP-treated plants. The expression of $H v E F 1 \alpha$ and $B S M V C P$ was detected at all time points. In contrast, the expression of $H \nu C E B i P$ was clearly down-regulated in $\mathrm{BSMV}: \mathrm{HvCEBiP}$-treated plants, confirming that $H \nu C E B i P$ had been silenced. The expression of $H v P A L$, $H v P R-2 a$ and $H v P R-5$ also appeared to be downregulated in BSMV:HvCEBiP-treated plants compared to BSMV-treated plants. However, the expression levels of $H v P R-1$ and $H v R B O H A$ in BSMV:HvCEBiP-treated plants were similar to those in BSMV-treated plants. These results suggest that the expression of $H v P A L$, $H v P R-2 a$ and $H v P R-5$ might be regulated by $H v C E B i P$ signaling.

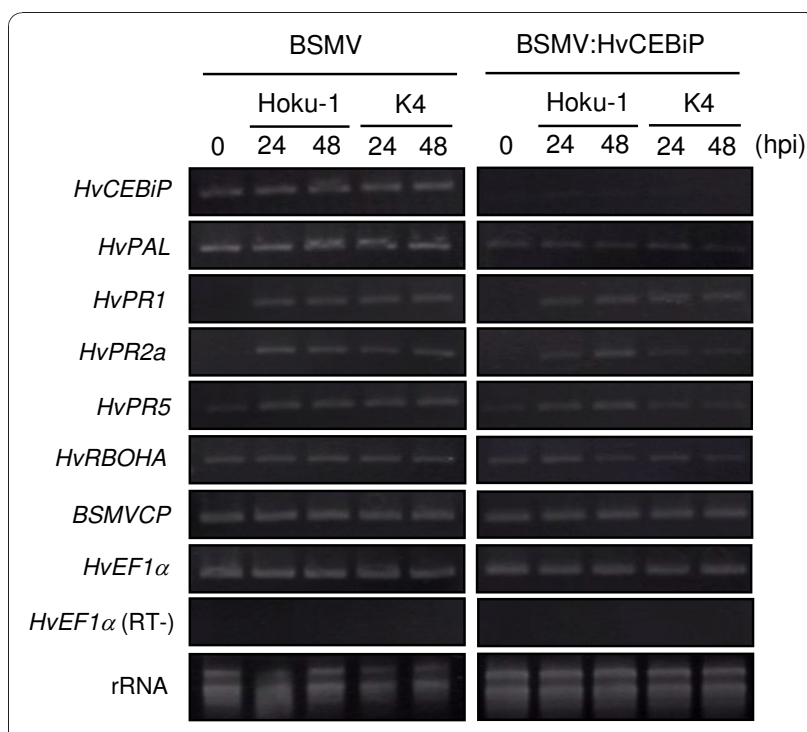

Figure 8 Expression profiling of defense-related genes in leaves of BSMV:HvCEBiP-treated plants. Total RNAs were extracted from the leaves of BSMV- or BSMV:HvCEBiP-treated plants inoculated with M. oryzae wild-type strain Hoku-1 or mossd 1 mutant $\mathrm{K} 4$ at 0 (no inoculation), 24 and 48 hpi. The expression of HVCEBiP was strongly down-regulated in BSMV:HVCEBiP-treated plants compared to BSMV-treated plants. The expression of HVPAL, HvPR-2a and HVPR-5 was also down-regulated in BSMV:HvCEBiP-treated plants compared to BSMV-treated plants. In contrast, the expression levels of HvPR-1 and HVRBOHA in BSMV:HvCEBiP-treated plants were similar to those in BSMV-treated plants.

\section{Discussion}

Barley expresses two layers of basal defense in response to infection by Magnaporthe oryzae

In our previous study, we generated an ssd 1 mutant of $M$. oryzae, in which the infection of rice plants was restricted by a defense response involving death of the initially infected epidermal cell [12]. This cell death reaction expressed by rice in response to compatible isolates of $M$. oryzae has been termed 'whole-plant specific resistance' (WPSR), and is independent of $R$-gene mediated resistance in rice $[23,24]$. In the present study, infection assays revealed that the mossd 1 mutant also showed attenuated pathogenicity on barley. However, the host defense responses expressed in barley to appressorial penetration by the mossd 1 mutant took the form of papilla deposition at attempted fungal entry sites rather than host cell death. The phenomenon of papilla formation during $M$. oryzae infection of barley has also been reported by other authors [18]. In rice, papilla-like wall appositions were also observed beneath appressoria of $M$. oryzae, although these appeared small and thin with electron microscopy [25]. Therefore, the formation of papillae appears to be a general form of basal defense against attempted appressorial penetration by $M$. oryzae in barley. However, the efficiency of 
papillae in restricting appressorial penetration seems to be weak because the wild-type strain could successfully penetrate into plant cells with high frequency, as shown in Figure 2C. Apart from papilla formation, a localized cell death reaction was also observed in the initially penetrated host cells in which infection hyphae had developed. This cell death reaction was observed in the leaves of BSMV:HvCEBiP-treated barley plants after infection by both the ssd 1 mutant and the wild-type strain of $M$. oryzae. The cell death reaction was associated with inhibition of fungal growth because infection hyphae had not developed beyond the first infected epidermal, even after 7 days. The barley cell death reaction resembles WPSR in rice [23] and conceivably it represents a basal defense response triggered after successful penetration by $M$. oryzae appressoria. It therefore appears that barley deploys two distinct layers of basal defenses against appressorium-mediated infection by $M$. oryzae, namely papilla formation and localized cell death. Two similar layers of plant defense were also shown to operate during non-host resistance of Arabidopsis to powdery mildew fungi [26].

\section{HvCEBiP is involved in basal resistance to appressorial penetration by $M$. oryzae}

In our recent work, we used the $C$. orbiculare ssd 1 mutant to show that a specific MAPK pathway in $N$. benthamiana plays a critical role in host basal defense but genes required for $R$-gene mediated resistance (RAR1, SGT1 and HSP90) do not [13]. Here, we used the $M$. oryzae ssd 1 mutant to examine the role in basal defense of genes required for penetration resistance and $R$-gene mediated resistance. Ror 1 and Ror 2 were identified as genes required for $m l o$-specific resistance against the barley powdery mildew fungus Blumeria graminis $\mathrm{f}$. sp. hordei and Ror 2 shows functional homology to syntaxin AtSYP121 in Arabidopsis [27]. Rar1 was originally shown to be required for race-specific resistance triggered by resistance gene Mla12 against B. graminis f. sp. hordei expressing the avirulence gene AvrMla12 [28,29]. Rom 1 was identified as a restoration of Mla12-specified resistance (rom1) mutant that restores disease resistance to $B$. graminis $\mathrm{f}$. sp. hordei carrying the avirulence gene AvrMla12 [22]. However, infectivity of the mossd1 mutant was not significantly enhanced on any of these barley mutants compared to wild-type plants, suggesting that genes required for $R$-gene mediated resistance do not play a role in basal defense against $M$. oryzae, consistent with findings from the C. orbiculare-N. benthamiana interaction [13].

In contrast to mutations in these barley genes, the knock-down of HvCEBiP did enhance infection by the mossd1 mutant. Thus, on BSMV:HvCEBiP-treated plants mutant K4 produced more severe (Type II) symptoms, i.e. brown necrotic flecks, compared to BMSV-treated control plants (Figure $5 \mathrm{~B}$ ). The silencing of $\mathrm{HvCEBiP}$ also increased the frequency of successful appressorial penetration by the mossd 1 mutant. However, the formation of infection hyphae inside penetrated epidermal cells appeared to trigger localized host cell death, resulting in brown necrotic symptoms. These results suggest that $H \nu C E B i P$ is involved in basal defense against appressorial penetration by $M$ oryzae. In contrast to the mossd 1 mutant, infectivity of the wild-type strain was not significantly enhanced on $H \nu C E B i P$-silenced plants but there was a slight increase in symptom severity. This suggests that although $H \nu C E B i P$ contributes to basal defense in barley, the level of its contribution may be low, so that with the highly pathogenic wild-type strain differences in symptoms between non-silenced and $H \nu C E B i P$-silenced plants were hard to distinguish. One plausible explanation of these findings is that basal defense against appressorial penetration involves multiple PAMP receptors and signaling pathways, of which signaling via HvCEBiP is only one. A working model for the contribution of $H \nu C E B i P$ to the dual-layered basal defense responses of barley to $M$. oryzae is presented in Figure 9.

In addition to the increased frequency of brown necrotic fleck symptoms induced by the mossd 1 mutant on BSMV:HvCEBiP-treated plants, a few inoculation sites also showed formation of severe blast lesions (Type III symptom) as shown in Figure 5A. Lesion formation was not associated with localized cell death reactions and infection hyphae developed extensively, colonizing many host cells. This suggests that in some cases the mossd 1 mutant was able to infect $H v C E B i P$-silenced plants without triggering cell death-associated defense responses. This raises the possibility that $H \nu C E B i P$ might be involved in mediating the localized cell death response of barley epidermal cells to invasion by $M$. oryzae infection hyphae. Thus, HvCEBiP might contribute not only to papilla-based defenses but also to the hypersensitive cell death response to cell invasion. $H \nu C E B i P$ does not appear to play a central role in nonhost resistance because the non-adapted pathogen C. graminicola produced no symptoms on silenced plants. In contrast, the LysM domain receptor kinase CERK1 was reported to contribute weakly to the resistance of Arabidopsis thaliana against the incompatible pathogen Alternaria brassicicola [30].

\section{Is HvCEBiP a specific receptor for components of the mossd1 mutant?}

In the interaction between cucumber anthracnose pathogen $C$. orbiculare and $N$. benthamiana, we reported previously that the altered fungal cell wall composition conferred by $s s d 1$ gene disruption triggers 


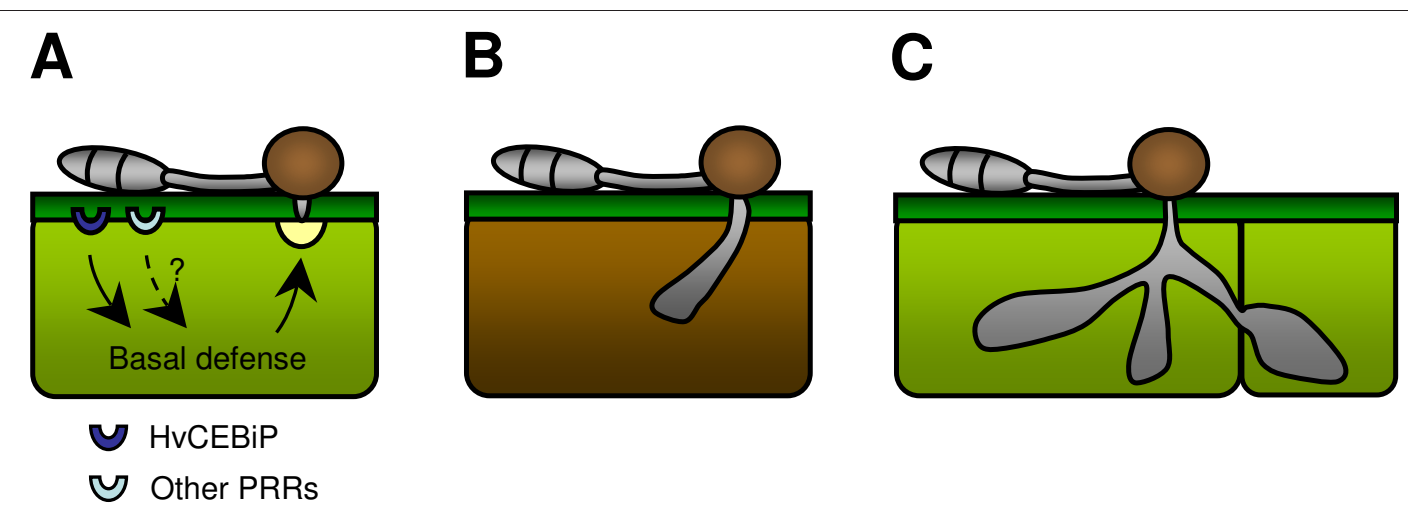

Figure 9 Working model for the involvement of HvCEBiP to dual layers basal defense in $M$. oryzae-barley interaction. (A) When an $M$. oryzae appressorium attempts to penetrate a barley epidermal cell, host basal defenses based on the formation of papillae are induced by the recognition of $M$. oryzae by HvCEBiP or other pattern recognition receptors (PRRs). However, this basal defense is insufficient to inhibit appressorial penetration by the wild-type strain, which successfully establishes infection hyphae inside living host cells. In contrast, appressorial penetration by the mossd1 mutant is effectively restricted by the formation of papillae at attempted entry sites. (B) When infection hyphae of the mossd 1 mutant successfully invade barley epidermal cells in HVCEBiP-silenced plants, a second layer of basal defense, associated with death of the initially infected cell, leads to restriction of hyphal development. This localized cell death also occurs in leaves inoculated with the wildtype strain, and may therefore be a general defense response to infection by M. oryzae. (C) When the wild-type strain successfully develops infection hyphae inside the initially infected cell without cell death reaction, the wild-type attempts the further infection to neighboring cells by development of infection hyphae.

plant basal resistance through the activation of a specific plant MAPK cascade [13]. We hypothesized that activation of the MAPK pathway might result from recognition of fungal PAMP(s) by corresponding plant receptor protein(s). In this study, we attempted to determine whether HvCEBiP is a specific receptor for PAMPs expressed uniquely by the mossd 1 mutant, in which case pathogenicity of the wild-type strain should not be affected by the silencing of $H \nu C E B i P$. However, the wild-type strain Hoku-1 showed a slight increase in pathogenicity on $H v C E B i P$-silenced plants, suggesting that HvCEBiP is a receptor for component(s) shared by both the wild-type $M$. oryzae and mossd 1 mutant.

Rice CEBiP is a receptor-like protein containing two LysM domains, which was originally identified in enzymes that degrade the bacterial cell wall component peptidoglycan [31]. Recent biochemical analysis showed that the LysM domain can also mediate binding to chitin oligosaccharides [32]. The genome of Arabidopsis contains five LysM domain-containing receptor-like kinases [33], among which CERK1 (At3g21630) was identified as a receptor-like protein required for chitin signaling in Arabidopsis [30]. Although the function of the other LysM domain-containing receptor-like kinases is unknown, it is tempting to speculate that plants possess multiple receptor proteins for the perception of particular classes of pathogen-derived oligosaccharides. It is likely that other PAMP receptors, in addition to HvCEBiP, are conserved in barley and contribute to basal resistance to $M$. oryzae.

\section{Conclusions}

Rice CEBiP recognizes chitin oligosaccharides derived from fungal cells leading to the expression of plant disease resistance against fungal infection. We evaluated the involvement of putative chitin receptor gene $\mathrm{H \nu CE}$ $B i P$ in barley basal resistance using the mossd 1 mutant of Magnaporthe oryzae, which enhances host basal defense responses. The mossd 1 mutant showed attenuated pathogenicity on barley and appressorial penetration was restricted by the formation of papillae at attempted entry sites. On $H \nu C E B i P$-silenced plants, the mutant produced small brown necrotic flecks or blast lesions accompanied by appressorium-mediated penetration into plant epidermal cells. Wild-type $M$. oryzae also produced slightly more severe symptoms on the leaves of $H \nu C E B i P$-silenced plants. These results indicated that $H \nu C E B i P$ is involved in basal resistance against appressorium-mediated infection and that basal resistance could be triggered by the recognition of chitin oligosaccharides derived from $M$. oryzae.

\section{Methods}

\section{Plant growth conditions and fungal strains}

Hordeum vulgare wild-type cultivars Fiber-snow, Ingrid and Sultan5, and genetic mutants mlo5, mlo5ror1, mlo5ror 2 , rar 1 and rom 1 were grown in a controlled environment chamber ( $16 \mathrm{~h}$ photoperiod, $24^{\circ} \mathrm{C}$ ). Magnaporthe oryzae Hoku-1 was used as the wild-type strain in this study. The mossd 1 mutants K1 and K4 were generated as reported previously [12]. These fungal cultures were maintained at $24^{\circ} \mathrm{C}$ on oatmeal agar medium $(6.0 \mathrm{~g}$ 
powder oatmeal, $1.25 \mathrm{~g}$ agar per $100 \mathrm{ml}$ distilled water) under continuous light. Colletotrichum graminicola isolate MAFF236902 was described previously [13].

\section{Pathogen inoculation and cytological assays}

To induce conidiation, two week-old cultures of $M$. ory$z a e$ were washed with sterile water to remove aerial hyphae and then incubated for a further 3 days. For inoculation, conidial suspension was sprayed $(5 \mathrm{ml} ; 1 \times$ $10^{6}$ conidia/ml $)$ or spotted $\left(10 \mu \mathrm{l} ; 1 \times 10^{5}\right.$ conidia $\left./ \mathrm{ml}\right)$ onto the third leaves of $H$. vulgare and incubated in a humid plastic box at $24^{\circ} \mathrm{C}$. For evaluation of invasive growth ability, the surface of barley leaves was scratched with a sterile plastic pipette tip and droplets of conidial suspensions were placed directly onto the wound sites. Cytological observations and the detection of papillae were performed as follows. Inoculated leaves were cut to $1 \mathrm{~cm} \times 1 \mathrm{~cm}$ size and decolorized with a 3:1 mixture of ethanol:chloroform and mounted under a coverslip in lactophenol solution. Autofluorescent papillae formed beneath appressoria were visualized by epifluoresence. The accumulation of $\mathrm{H}_{2} \mathrm{O}_{2}$ in host cells was detected by staining with 3,3'-diaminobenzidine [13].

\section{RT-PCR}

Total RNA was extracted from barley leaves using TRIzol Reagent (Invitrogen) following the manufacturer's protocol. RT-PCR was performed using ReverTra Dash RT-PCR kit (Toyobo) following the manufacturer's protocol. The primers used for RT-PCR are listed in Additional file 1: Table S1. The sequence data of $H v P A L$, $H v P R-1, H v P R-2 a, H v P R-5, H v R B O H A$ and HvEF1 $\alpha$ can be found in GeneBank with accession numbers Z49147, Z21494, AY612193, AF355455, AJ871131 and Z50789, respectively.

\section{Vector construction}

A 298 bp partial fragment of $H \nu C E B i P$ was amplified by primer pairs HvCBP1-S1 (5'-CCAAAGACCCTCAAGAAGGA-3') and HvCBP1-AS1 (5'-AGCCGTTGGAATAACCACTG-3') from cDNA of $H$. vulgare and subcloned into the pGEM-T easy vector (Promega). The resulting construct was digested by NotI and a fragment containing the amplified sequence of $H \nu C E B i P$ was introduced into the NotI site of pSL038-1 in the antisense orientation. This construct was designated as $\mathrm{p} \gamma$ : HvCEBiPas.

\section{Virus-induced gene silencing}

BSMV genomic RNAs were transcribed in vitro as previously described with some modifications [17]. The reaction was performed at $37^{\circ} \mathrm{C}$ for $60 \mathrm{~min}$ in $50 \mu \mathrm{l}$ of reaction buffer containing $1 \mu \mathrm{g}$ of linearized plasmids, 1 $\mu \mathrm{l}$ of T7 RNA polymerase (Takara), $10 \mu \mathrm{l}$ of $50 \mathrm{mM}$
DTT, $6 \mu \mathrm{l}$ of $10 \mathrm{mM}$ NTPs (rATP, rCTP, rUTP), $0.4 \mu \mathrm{l}$ of $10 \mathrm{mM}$ rGTP and $5 \mu \mathrm{l}$ of $5 \mathrm{mM} \mathrm{m}^{7} \mathrm{G}$ (ppp)G RNA cap structure analog (New England Biolabs). After the reaction, $1.62 \mu \mathrm{l}$ of $10 \mathrm{mM}$ rGTP and $1 \mu \mathrm{l}$ of T7 RNA polymerase were added to the reaction mixture, and further incubated at $37^{\circ} \mathrm{C}$ for $60 \mathrm{~min}$. Transcribed $\alpha, \beta$, $\gamma$ genomic RNAs were mixed in a 1:1:1 ratio with $20 \mu \mathrm{l}$ FES and inoculated onto the first-developed leaves of $H$. vulgare plants with gentle rubbing. The third-developed leaves were used for evaluating fungal infections.

\section{Additional material}

Additional file 1: Figure S1. Efficiency of BSMV-mediated gene silencing in barley. (A) photobleaching by gene silencing of phytoene desaturase (PDS) in barley. BSMV:PDS was inoculated onto the first developed leaf (1). After 10 days, photobleacing was observed in the third developed leaf (3). (B) close-up photograph of third- and fourth- developed leaves shown in A. (C) photobleaching phenotypes in five individual plants treated with BSMV:PDS. Third leaves of all five plants showed photobleaching. Table S1. Primers used for RT-PCR.

\section{Acknowledgements}

We are grateful to Dr. Kazuyuki Mise (Kyoto University) for technical advice about in vitro transcription. We are grateful to Dr. Steve Scofield (USDA-ARS, West Lafayette) for providing BSMV vectors and Professor Paul Schulze-Lefert (Max Planck Institute for Plant Breeding Research) for providing barley mutant seeds. This work was supported by Grants-in-Aid for Scientific Research from the Ministry of Education, Culture, Sports, Science and Technology (No.19380029 and 21380031) and JSPS Fellowships from the Ministry of Education, Culture, Sports, Science and Technology (No. 19380024)

\section{Author details}

${ }^{1}$ Laboratory of Plant Pathology, Graduate School of Life and Environmental Sciences, Kyoto Prefectural University, Kyoto 606-8522, Japan. ${ }^{2}$ Advanced Science Research Center, Kanazawa University, Ishikawa 920-0934, Japan. ${ }^{3}$ Department of Bioproduction Sciences, Ishikawa Prefectural University, Ishikawa 921-8836, Japan. ${ }^{4}$ Division of Plant Sciences, National Institute of Agrobiological Sciences, Ibaraki 305-8602, Japan. ${ }^{5}$ Department of Plant Microbe Interactions, Max Planck Institute for Plant Breeding Research, Carl von Linné Weg 10, D-50829 Köln, Germany. 'Department of Organismic Interactions, Max Planck Institute for Terrestrial Microbiology. Karl-von-FrischStrasse 35043 Marburg, Germany.

\section{Authors' contributions}

ST designed the experiments, performed the gene silencing study and wrote the manuscript. Al performed the sample preparations and vector construction. KY performed the inoculation assay for barley mutant lines. GT participated in experimental procedures for PCR analysis. HK participated in cytological analysis of barley infection assay. MM participated in barley gene silencing and data analysis, TN participated in barley infection assay and data analysis. NY participated in experimental procedures concerning CEBiP and data analysis. $\mathrm{RO}$ supervised the study and critically revised the manuscript. YK conceived and directed the whole study, and participated in the writing of the manuscript. All authors read and approved the final manuscript.

Received: 9 May 2010 Accepted: 30 December 2010 Published: 30 December 2010

\section{References}

1. Thordal-Christensen $\mathrm{H}$ : Fresh insights into processes of nonhost resistance. Current Opinion in Plant Biology 2003, 6:351-357. 
2. Nurnberger $T$, Brunner $F$, Kemmerling B, Piater L: Innate immunity in plants and animals: striking similarity and obvious differences. Immunological Reviews 2004, 198:249-266.

3. Jones JD, Dangl JL: The plant immune system. Nature 2006, 444:323-329.

4. Felix G, Duran JD, Volko S, Boller T: Plants have a sensitive perception system for the most conserved domain of bacterial flagellin. Plant Journal 1999, 18:265-276

5. Gómez-Gómez L, Bauer Z, Boller T: Both the extracellular leucine-rich repeat domain and the kinase activity of FSL2 are required for flagellin binding and signaling in Arabidopsis. Plant Cell 2001, 13:1155-1163.

6. Kunze G, Zipfel C, Robatzek S, Niehaus K, Boller T, Felix G: The N terminus of bacterial elongation factor Tu elicits innate immunity in Arabidopsis plants. Plant Cell 2004, 16:3496-3507.

7. Zipfel C, Kunze G, Chinchilla D, Caniard A, Jones JD, Boller T, Felix G Perception of the bacterial PAMP EF-Tu by the receptor EFR restricts Agrobacterium-mediated transformation. Cell 2006, 125:749-760.

8. Zhang B, Ramonell K, Somerville S, Stacey G: Characterization of early, chitin-induced gene expression in Arabidopsis. Molecular Plant-Microbe Interactions 2002, 15:963-970.

9. Shinya T, Ménard R, Kozone I, Matsuoka H, Shibuya N, Kauffmann S, Matsuoka K, Saito M: Novel beta-1,3-, 1,6-oligoglucan elicitor from Alternaria alternata 102 for defense responses in tobacco. FEBS Journal 2006, 273:2421-2431.

10. Kaku H, Nishizawa Y, Ishii-Minami N, Akimoto-Tomiyama C, Dohmae N, Takio K, Minami E, Shibuya N: Plant cells recognize chitin fragments for defense signaling through a plasma membrane receptor. Proceedings of National Academy of Sciences USA 2006, 103:11086-11091.

11. Wilson RA, Talbot NJ: Under pressure: investigating the biology of plant infection by Magnaporthe oryzae. Nature Reviews Microbiology 2009, 7:185-195.

12. Tanaka S, Yamada K, Yabumoto K, Fujii S, Huser A, Tsuji G, Koga H, Dohi K, Mori M, Shiraishi T, O'Connell R, Kubo Y: Saccharomyces cerevisiae SSD1 orthologues are essential for host infection by the ascomycete plant pathogens Colletotrichum lagenarium and Magnaporthe grisea. Molecular Microbiology 2007, 64:1332-1349.

13. Tanaka S, Ishihama N, Yoshioka H, Huser A, O'Connell R, Tsuji G, Tsuge S, Kubo Y: The Colletotrichum orbiculare ssd 1 mutant enhances Nicotiana benthamiana basal resistance by activating a mitogen-activated protein kinase pathway. Plant Cell 2009, 21:2517-2526.

14. Wesley SV, Helliwell CA, Smith NA, Wang MB, Rouse DT, Liu Q, Gooding PS, Singh SP, Abbott D, Stoutjesdijk PA, Robinson SP, Gleave AP, Green AG, Waterhouse PM: Construct design for efficient, effective and highthroughput gene silencing in plants. Plant Journal 2001, 27:581-590.

15. Ruiz MT, Voinnet O, Baulcombe DC: Initiation and maintenance of virusinduced gene silencing. Plant Cell 1998, 10:937-946.

16. Holzberg S, Brosio P, Gross C, Pogue GP: Barley stripe mosaic virus induced gene silencing in a monocot plant. Plant Journal 2002, 30:315-327.

17. Scofield SR, Huang L, Brandt AS, Gill BS: Development of a virus-induced gene-silencing system for hexaploid wheat and its use in functional analysis of the Lr21-mediated leaf rust resistance pathway. Plant Physiology 2005, 138:2165-2173

18. Jarosch B, Collins NC, Zellerhoff N, Schaffrath U: RAR1, ROR1, and the actin cytoskeleton contribute to basal resistance to Magnaporthe grisea in barley. Molecular Plant-Microbe Interactions 2005, 18:397-404.

19. Freialdenhoven A, Peterhansel $C$, Kurth J, Kreuzaler F, Schulze-Lefert $P$ : Identification of genes required for the function of non-race-specific $\mathrm{mlo}$ resistance to powdery mildew in barley. Plant Cell 1996, 8:5-14.

20. Collins NC, Lahaye T, Peterhänsel C, Freialdenhoven A, Corbitt M, SchulzeLefert P: Sequence haplotypes revealed by sequence-tagged site fine mapping of the Ror1 gene in the centromeric region of barley chromosome 1H. Plant Physiology 2001, 125:1236-1247.

21. Shirasu K, Lahaye T, Tan MW, Zhou F, Azevedo C, Schulze-Lefert P: A novel class of eukaryotic zinc-binding proteins is required for disease resistance signaling in barley and development in C. elegans. Cell 1999, 99:355-366.

22. Freialdenhoven A, Orme J, Lahaye T, Schulze-Lefert P: Barley Rom1 reveals a potential link between race-specific and nonhost resistance responses to powdery mildew fungi. Molecular Plant-Microbe Interactions 2005, 18:291-299.
23. Koga H, Dohi K, Mori M: Abscisic acid and low temperatures suppress the whole plant-specific resistance reaction of rice plants to the infection of Magnaporthe grisea. Physiological and Molecular Plant Pathology 2004, 65:3-9.

24. Koga H, Dohi K, Yoshimoto R, Mori M: Resistance in leaf blades assessed by counting conidia correlates with whole-plant-specific resistance in leaf sheaths in a compatible rice-Magnaporthe oryzae interaction. Journal of General Plant Pathology 2008, 74:246-249.

25. Koga $\mathrm{H}$ : Hypersensitive death, autofluorescence, and ultrastructural changes in cells of leaf sheaths of susceptible and resistant nearisogenic lines of rice $\left(P i-z^{t}\right)$ in relation to penetration and growth of Pyricularia oryzae. Canadian Journal of Botany 1994, 72:1463-1477.

26. Lipka V, Dittgen J, Bednarek P, Bhat R, Wiermer M, Stein M, Landtag J, Brandt W, Rosahl S, Scheel D, Llorente F, Molina A, Parker J, Somerville S, Schulze-Lefert P: Pre- and postinvasion defenses both contribute to nonhost resistance in Arabidopsis. Science 2005, 301:1180-1183.

27. Collins NC, Thordal-Christensen H, Lipka V, Bau S, Kombrink E, Qiu JL, Hückelhoven R, Stein M, Freialdenhoven A, Somerville SC, Schulze-Lefert P: SNARE-protein-mediated disease resistance at the plant cell wall. Nature 2003, 425:973-977.

28. Freialdenhoven A, Scherag B, Hollricher K, Collinge D, Christensen HT, Schulze-Lefert P: Rar-1 and Rar-2, two loci required for Mla12-specified race-specific resistance to powdery mildew in barley. Plant Cell 1994, 6:983-994.

29. Shen Q, Zhou F, Bieri S, Haizel T, Shirasu K, Schulze-Lefert P: Recognition specificity and RAR1/SGT1 dependence in barley Mla disease resistance genes to the powdery mildew fungus. Plant Cell 2003, 15:732-744.

30. Miya A, Albert P, Shinya T, Desaki Y, Ichimura K, Shirasu K, Narusaka Y, Kawakami N, Kaku H, Shibuya N: CERK1, a LysM receptor kinase, is essential for chitin elicitor signaling in Arabidopsis. Proceedings of National Academy of Sciences USA 2007, 104:19613-19618.

31. Joris B, Englebert $S$, Chu CP, Kariyama R, Daneo-Moore L, Shockman GD, Ghuysen JM: Modular design of the Enterococcus hirae muramidase-2 and Streptococcus faecalis autolysin. FEMS Microbiology Letters 1992, 70:257-264.

32. Ohnuma T, Onaga S, Murata K, Taira T, Katoh E: LysM domains from Pteris ryukyuensis chitinase-A: a stability study and characterization of the chitin-binding site. Journal of Biological Chemistry 2008, 283:5178-5187.

33. Wan J, Zhang XC, Neece D, Ramonell KM, Clough S, Kim SY, Stacey MG, Stacey G: A LysM receptor-like kinase plays a critical role in chitin signaling and fungal resistance in Arabidopsis. Plant Cell 2008, 20:471-481.

\section{doi:10.1186/1471-2229-10-288}

Cite this article as: Tanaka et al: HvCEBiP, a gene homologous to rice chitin receptor CEBiP, contributes to basal resistance of barley to Magnaporthe oryzae. BMC Plant Biology 2010 10:288.

\section{Submit your next manuscript to BioMed Central and take full advantage of:}

- Convenient online submission

- Thorough peer review

- No space constraints or color figure charges

- Immediate publication on acceptance

- Inclusion in PubMed, CAS, Scopus and Google Scholar

- Research which is freely available for redistribution

Submit your manuscript at www.biomedcentral.com/submit
C Biomed Central 